\title{
Hölder stability for Serrin's overdetermined problem
}

\author{
Giulio Ciraolo $^{1}$ - Rolando Magnanini ${ }^{2}$. \\ Vincenzo Vespri ${ }^{2}$
}

Received: 10 February 2015 / Accepted: 22 June 2015 / Published online: 8 July 2015

(C) Fondazione Annali di Matematica Pura ed Applicata and Springer-Verlag Berlin Heidelberg 2015

\begin{abstract}
In a bounded domain $\Omega$, we consider a positive solution of the problem $\Delta u+$ $f(u)=0$ in $\Omega, u=0$ on $\partial \Omega$, where $f: \mathbb{R} \rightarrow \mathbb{R}$ is a locally Lipschitz continuous function. Under sufficient conditions on $\Omega$ (for instance, if $\Omega$ is convex), we show that $\partial \Omega$ is contained in a spherical annulus of radii $r_{i}<r_{e}$, where $r_{e}-r_{i} \leq C\left[u_{\nu}\right]_{\partial \Omega}^{\tau}$ for some constants $C>0$ and $\tau \in(0,1]$. Here, $\left[u_{\nu}\right]_{\partial \Omega}$ is the Lipschitz seminorm on $\partial \Omega$ of the normal derivative of $u$. This result improves to Hölder stability the logarithmic estimate obtained in Aftalion et al. (Adv Differ Equ 4:907-932, 1999) for Serrin's overdetermined problem. It also extends to a large class of semilinear equations the Hölder estimate obtained in Brandolini et al. ( $\mathrm{J}$ Differ Equ 245:1566-1583, 2008) for the case of torsional rigidity ( $f \equiv 1)$ by means of integral identities. The proof hinges on ideas contained in Aftalion et al. (1999) and uses Carleson-type estimates and improved Harnack inequalities in cones.
\end{abstract}

Keywords Serrin's problem · Overdetermined problems - Method of moving planes · Stability $\cdot$ Stationary surfaces $\cdot$ Harnack's inequality

Mathematics Subject Classification Primary 35B06 - 35J05 · 35J61 - Secondary 35B35 . 35B09

Giulio Ciraolo
giulio.ciraolo@unipa.it
http://www.math.unipa.it/ g.ciraolo/
Rolando Magnanini
magnanin@ math.unifi.it
http://web.math.unifi.it/users/magnanin
Vincenzo Vespri
vespri@math.unifi.it
http://web.math.unifi.it/users/vespri
Dipartimento di Matematica e Informatica, Università di Palermo,
Via Archirafi 34, 90123 Palermo, Italy
Dipartimento di Matematica ed Informatica “U. Dini”, Università di Firenze,
viale Morgagni 67/A, 50134 Florence, Italy 


\section{Introduction}

Serrin's overdetermined problem has been the object of many investigations. In its classical form, it involves a sufficiently smooth bounded domain $\Omega$ in $\mathbb{R}^{N}$ and a classical solution of the set of equations:

$$
\begin{aligned}
\Delta u+f(u) & =0 \text { and } u \geq 0 \text { in } \Omega, \quad u=0 \text { on } \partial \Omega, \\
u_{v} & =c \text { on } \partial \Omega .
\end{aligned}
$$

Here, $f:[0,+\infty) \rightarrow \mathbb{R}$ is a locally Lipschitz continuous function, $u_{v}$ denotes the inward normal derivative of $u$, and $c$ is a positive constant. Under these assumptions, Serrin [24] proved that $\Omega$ must be a ball and $u$ be radially symmetric. For his proof, he adapted and improved a method created by Aleksandrov to prove his Soap Bubble Theorem (see [2]). In fact, the radial symmetry of $\Omega$ and $u$ is obtained by the so-called method of moving planes based upon the observation that the Euclidean ball is the only bounded domain that is symmetric with respect to any hyperplane passing through its center of mass. There are other interesting proofs of this symmetry result, based on integral identities, that generally need severe restrictions on $f$ (see for instance $[5,23,26]$ ).

Overdetermined problems such as (1.1)-(1.2) arise in many physical and geometric situations; they can be seen as a prototype of inverse problems and often emerge in free boundary and shape optimization problems (see [14]).

Despite the intense research that has been devoted to them for almost five decades, there are still many open problems. An important one - the focus of this paper-concerns the study of the stability of the radial configuration.

The first contribution in this direction is [1], where it is proved that if one assumes that $u_{v}$ is almost constant on $\partial \Omega$, then there exist two concentric balls $B_{r_{i}}$ and $B_{r_{e}}$, with

$$
B_{r_{i}} \subset \Omega \subset B_{r_{e}}
$$

and such that $r_{e}-r_{i}$ can be bounded in terms of some measure of the deviation of $u_{v}$ from being a constant. More precisely, in [1] it is proved the estimate

$$
r_{e}-r_{i} \leq C\left|\log \left\|u_{v}-d\right\|_{C^{1}(\partial \Omega)}\right|^{-1 / N},
$$

where $d$ is some given constant, provided $\left\|u_{v}-d\right\|_{C^{1}(\partial \Omega)}$ is sufficiently small; here, $C$ is a constant depending on $N$, the regularity of $\partial \Omega$, the diameter of $\Omega$ and the Lipschtz constant of $f$. The proof is based on a quantitative study of the method of moving planes and works for a general locally Lipschitz nonlinearity $f$.

In the case of the torsional rigidity problem, that is when $f \equiv 1,(1.4)$ was improved in [6] (see also [7] for Monge-Ampère equations). Indeed, the authors replace the logarithmic dependence at the right-hand side of (1.4) by a power law of Hölder type. Furthermore, they also give a stability estimate in terms of the $L^{1}$-norm of the deviation instead of its $C^{1}$-norm.

In our main result, Theorem 2.4, we will show that the logarithmic estimate (1.4) can be improved to obtain a stability of Hölder type. In order to avoid unnecessary technicalities, in this section we present our result in a relevant particular case. In what follows, we denote by $d_{\Omega}$ the diameter of $\Omega, r_{\Omega}$ the radius of the optimal interior touching sphere to $\partial \Omega$ (see Sect. 2 for its precise definition), and use the following notation:

$$
\left[u_{v}\right]_{\partial \Omega}=\sup _{\substack{x, y \in \partial \Omega \\ x \neq y}} \frac{\left|u_{v}(x)-u_{v}(y)\right|}{|x-y|} .
$$


Theorem 1.1 Let $\Omega \subset \mathbb{R}^{N}$ be a convex domain with boundary of class $C^{2, \alpha}$ and let $f$ be a locally Lipschitz continuous function such that $f(0) \geq 0$. Let $u \in C^{2, \alpha}(\bar{\Omega})$ be a solution of (1.1).

There exist two positive numbers $\varepsilon, C$ that depend on

$$
N, f, d_{\Omega}, r_{\Omega} \text {, the } C^{2, \alpha} \text {-regularity of } \Omega, \max _{\bar{\Omega}} u, \min _{\partial \Omega} u_{v},
$$

and a number $\tau \in(0,1)$ such that, if $\left[u_{\nu}\right]_{\partial \Omega} \leq \varepsilon$, then

$$
B_{r_{i}} \subset \Omega \subset B_{r_{e}},
$$

where $B_{r_{i}}$ and $B_{r_{e}}$ are two concentric balls and their radii satisfy

$$
r_{e}-r_{i} \leq C\left[u_{\nu}\right]_{\partial \Omega}^{\tau}
$$

The number $\tau$ can be explicitly determined (see Theorem 2.4, for details). Notice that the seminorm (1.5) can be bounded in terms of the deviation used in (1.4), if we choose $d$ as the minimum of $u_{v}$ on $\partial \Omega$.

Therefore, (1.6) significantly improves the estimates of [1] and [6], since it enhances the stability from logarithmic, as in (1.4), to that of Hölder type proved in [6], but for any locally Lipschitz nonlinearity $f$ (that is, not only for the case $f \equiv 1$ ).

The assumption on the convexity of $\Omega$ can be slightly relaxed (see Theorem 2.4), by requiring that $\Omega$ be what we call a $(\mathcal{C}, \theta)$-domain. Roughly speaking, we require that every maximal cap that comes about in employing the method of moving planes has a boundary with a Lipschitz constant bounded uniformly with respect to the direction of mirror reflection chosen (see Sect. 2 for details).

As already mentioned, our results and the ones in [6] are obtained by using very different techniques. The starting point of [6] is the proof of symmetry given by the same authors in [5] which makes use of information, such as Newton inequalities and Pohozaev identity, which is in a sense more global and avoids an extensive use of maximum principles needed to employ the method of moving planes. On the other hand, the method in [6] is more restrictive, since it seems to be suitable only for $f$ constant.

Instead, based on Serrin's original proof, our approach is more flexible and allows to treat a general Lipschitz continuous nonlinearity $f$; in fact, our study relies on quantitative versions of the maximum principle, such as (global) Harnack-type inequalities. Thus, the quality of the relevant stability estimate is affected by that of the Harnack inequality we employ. This is the reason why, at this stage, even if we can consider a general nonlinearity, we need to put a restriction on the type of domain under study. In particular, for convex and $(\mathcal{C}, \theta)$-domains, we can use improved Harnack-type inequalities in every cap which is generated by the method of moving planes.

Besides by those in [1], the techniques used in this paper are inspired by those employed in [11] (see also [10]), where a quantitative study of the radially symmetric configuration was carried out for a related problem - the parallel surface problem - motivated by the remark, made in [19] (see also [18,20]), that time-invariant level surfaces of solutions of certain nonlinear non-degenerate fast diffusion equations are parallel surfaces.

As in [1] and [11], our approach consists in fixing a direction, defining an approximate set $X(\delta)$-built upon the so-called maximal cap (see Sect. 2) and its mirror-symmetric image in that direction-which fits $\Omega$ well, as the parameter $\delta$ tends to 0 . This approximation process is controlled in terms of $\left[u_{\nu}\right]_{\partial \Omega}$ and does not depend on the particular direction chosen.

The application of Harnack's inequality and Carleson estimates in the maximal cap plays a crucial role in obtaining the new stability estimates. Since we are assuming that every 
maximal cap has Lipschitz regularity, the improvement in (1.6) is obtained by a refinement of Harnack's inequality in suitable cones.

The paper is organized as follows. In Sect. 2, we recall the notations and preliminaries necessary to use the method of moving planes. There, we also discuss on the definition of $(\mathcal{C}, \theta)$-domains and present our main result, Theorem 2.4 , of which Theorem 1.1 is a straightforward corollary. In Sect. 3-the core of the paper-we begin the proof of Theorem 2.4, by producing the necessary enhanced Harnack estimates. Finally, in Sect. 4, we complete the proof of (1.6).

\section{Remarks on the method of moving planes and statement of the main result}

We consider a bounded domain $\Omega$ of class $C^{2, \alpha}, 0<\alpha \leq 1$. In what follows, we will often use the notation $C\left(N, \Omega, d_{\Omega}, r_{\Omega}, f, \ldots\right)$ to denote a constant that depends on the relevant parameters; in particular, the dependence on $\Omega$ is meant to be only on the $C^{2, \alpha}$ regularity of $\partial \Omega$, as explained in [1, Remark 1]. The assumed regularity of $\Omega$ implies that $\Omega$ satisfies a uniform interior sphere condition; for $x \in \partial \Omega$, we let $r(x)$ be the radius of the maximal ball $B \subset \Omega$ with $x \in \partial B$ and set

$$
r_{\Omega}=\min _{x \in \partial \Omega} r(x) .
$$

In the sequel, it will be useful to consider the parallel set of $\Omega$ :

$$
\Omega(\delta)=\{x \in \Omega: \operatorname{dist}(x, \partial \Omega)>\delta\} .
$$

If $0<\delta<r_{\Omega}, \partial \Omega(\delta)$ is $C^{2, \alpha}$-smooth; also, $\Omega(\delta)$ satisfies a uniform interior sphere condition with optimal radius $r_{\Omega}-\delta$.

For a unit vector $\omega \in \mathbb{R}^{N}$ and a parameter $\mu \in \mathbb{R}$, we define the following objects:

$$
\begin{array}{ll}
\pi_{\mu}=\left\{x \in \mathbb{R}^{N}: x \cdot \omega=\mu\right\} & \text { a hyperplane orthogonal to } \omega, \\
\mathcal{H}_{\mu}=\left\{x \in \mathbb{R}^{N}: x \cdot \omega>\mu\right\} & \text { the half-space on the right of } \pi_{\mu}, \\
\Omega_{\mu}=\{x \in A: x \cdot \omega>\mu\} & \text { the right-hand cap of } \Omega, \\
x^{\mu}=x-2(x \cdot \omega-\mu) \omega & \text { the reflected image of } x \text { in } \pi_{\mu}, \\
\Omega^{\mu}=\left\{x \in \mathbb{R}^{N}: x^{\mu} \in \Omega_{\mu}\right\} & \text { the reflected cap in } \pi_{\mu} .
\end{array}
$$

Set $\Lambda=\sup \{x \cdot \omega: x \in \Omega\}$, the extent of $\Omega$ in direction $\Omega$; if $\mu<\Lambda$ is close to $\Lambda$, the reflected cap $\Omega^{\mu}$ is contained in $\Omega$ (see [12]). Set

$$
\lambda=\inf \left\{\mu: \Omega^{\mu^{\prime}} \subset \Omega \text { for all } \mu^{\prime} \in(\mu, \Lambda)\right\} .
$$

Then, at least one of the following cases occurs (see [12,24]):

(S1) $\Omega^{\lambda}$ becomes tangent to $\partial \Omega$ at some point $P^{\lambda} \in \partial \Omega^{\lambda} \backslash \pi_{\lambda}$, that is the reflected image of a point $P \in \partial \Omega_{\lambda} \backslash \pi_{\lambda}$;

(S2) $\pi_{\lambda}$ is orthogonal to $\partial \Omega$ at some point $Q \in \partial \Omega \cap \pi_{\lambda}$.

The cap $\Omega_{\lambda}$ will be called the maximal cap.

As customary in the method of moving planes, we define the function

$$
w(x)=u\left(x^{\lambda}\right)-u(x), \quad x \in \Omega_{\lambda} ;
$$

$w$ satisfies the equation

$$
\Delta w+c(x) w=0 \quad \text { in } \Omega_{\lambda},
$$


where for $x \in \Omega_{\lambda}$

$$
c(x)= \begin{cases}\frac{f\left(u\left(x^{\lambda}\right)\right)-f(u(x))}{u\left(x^{\lambda}\right)-u(x)} & \text { if } u\left(x^{\lambda}\right) \neq u(x), \\ 0 & \text { if } u\left(x^{\lambda}\right)=u(x) .\end{cases}
$$

Notice that $c(x)$ is bounded by the Lipschitz constant $\mathfrak{L}_{f}$ of $f$ in the interval $\left[0, \max _{\bar{\Omega}} u\right]$.

All the improved estimates in Sect. 3 concern $w$. As proved in [24] and refined in [4] (see also [12]), since $w \geq 0$ on $\partial \Omega_{\lambda}$, we can assume that $w \geq 0$ in $\Omega_{\lambda}$. Hence, a standard application of the strong maximum principle to the inequality $\Delta w-c^{-}(x) w \leq 0$ with $c^{-}(x)=\max [-c(x), 0]$ shows that either $w=0$ in $\Omega_{\lambda}$ (and $\Omega$ and $u$ are symmetric about $\pi_{\lambda}$ ) or

$$
w>0 \text { in } \Omega_{\lambda}
$$

The following lemma ensures that the maximal cap always contains a half-ball tangent to $\partial \Omega$ at either point $P$ or $Q$.

Lemma 2.1 Let $P$ and $Q$ be as in case (S1) and (S2), respectively. Let $B_{\rho}(p) \subset \Omega$ be a ball with $0<\rho \leq r_{\Omega}$ and such that $P \in \partial B_{\rho}(p)$ or $Q \in \partial B_{\rho}(p)$.

Then, $p \in \bar{\Omega}_{\lambda}$ and $B_{\rho}(p) \cap \mathcal{H}_{\lambda} \subset \Omega_{\lambda}$.

Proof The assertion is trivial for case (S2).

If case (S1) occurs, without loss of generality, we can assume that $\omega=e_{1}=(1,0, \ldots, 0)$ and $\lambda=0$. Since (S1) holds, the point $P^{\lambda}$ lies on $\partial \Omega$ and cannot fall inside $B_{\rho}(p)$, since $P \in \partial B_{\rho}(p)$ and $B_{\rho}(p) \subset \Omega$. Thus, $\left|p-P^{\lambda}\right| \geq \rho=|p-P|$ and hence $\left|p_{1}+P_{1}\right| \geq\left|p_{1}-P_{1}\right|$, which implies that $p_{1} \geq 0$, being $P_{1}>0$.

As mentioned in the Introduction, the convexity assumption of Theorem 1.1 can be relaxed; with this purpose, we introduce the class of $(\mathcal{C}, \theta)$-domains that, roughly speaking, have the property that every maximal cap $\Omega_{\lambda}$ has a boundary with a Lipschitz constant bounded by a number which does not depend on the direction of reflection chosen. To this aim, for a fixed $0<t<1 / 2$, by formula (2.1), we define the set

$$
G=\Omega\left(t r_{\Omega}\right)
$$

we know that $G$ is connected (see [1, pp. 923-924]). Thus, we say that $\Omega$ is a $(\mathcal{C}, \theta)$-domain if there exists $\theta>0$ such that for any direction $\Omega$ and for any $x \in \Omega_{\lambda} \backslash \bar{G}_{\lambda}$, there exists $\xi \in \partial G_{\lambda} \backslash \pi_{\lambda}$ such that $x$ and $\xi$ belong to the axis of a (finite) right spherical cone $\mathcal{C} \subset \Omega_{\lambda}$ with vertex at $x$ and aperture $2 \theta$.

This property is not easy to check, since it relies on the knowledge of the position of the critical hyperplane (see Fig. 1). However, the class of $(\mathcal{C}, \theta)$-domains is not empty, as shown by the following proposition.

Proposition 2.2 Any convex domain $\Omega$ of class $C^{1}$ and satisfying the uniform interior sphere condition with minimal radius $r_{\Omega}$ is a $(\mathcal{C}, \theta)$-domain with

$$
\theta \geq \arctan \frac{(1-t) r_{\Omega}}{2 d_{\Omega}}
$$

where $t$ is the parameter appearing in (2.6). 

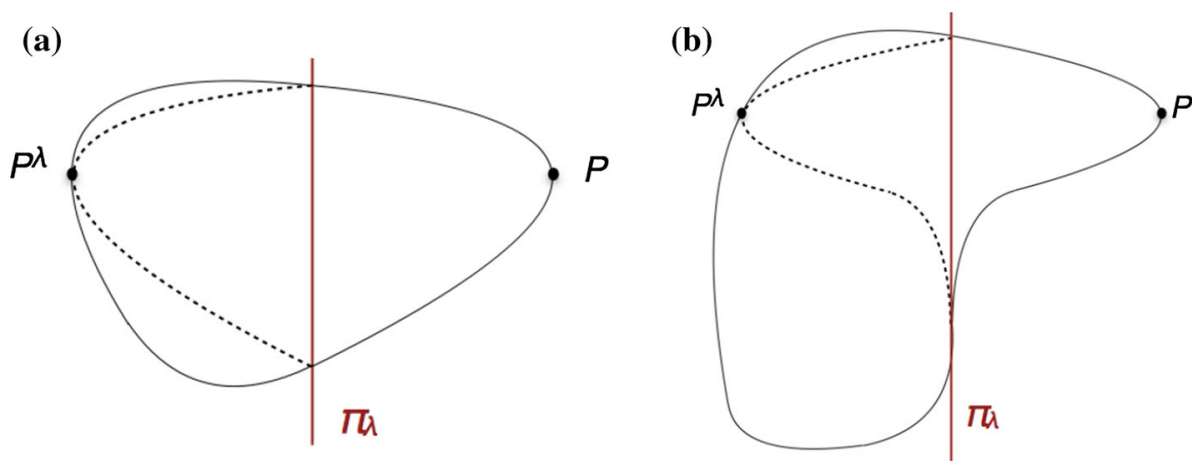

Fig. 1 Every maximal cap of a $(\mathcal{C}, \theta)$-domain has Lipschitz boundary. a A $(\mathcal{C}, \theta)$-domain. b Not a $(\mathcal{C}, \theta)$ domain

Proof Since $\Omega$ is of class $C^{1}$, then the method of moving planes can be applied (see [12]). As already observed, $G$ satisfies a uniform interior sphere condition with optimal radius $(1-t) r_{\Omega} ;$ also,

$$
\Omega=\left\{x+y: x \in G,|y|<t r_{\Omega}\right\},
$$

and $G, G_{\lambda}$ and $\Omega_{\lambda}$ are all convex, since $\Omega$ is convex.

From Lemma 2.1, we have that $\Omega_{\lambda}$ contains a half-ball of radius $r_{\Omega}$ with center $p \in \bar{\Omega}_{\lambda}$, and $G_{\lambda}$ contains the half-ball of radius $(1-t) r_{\Omega}$ centered at $p$. The maximal ball $B$ contained in this half-ball has radius $\bar{r}=(1-t) r_{\Omega} / 2$ and is contained in $G_{\lambda}$; we denote its center by $y$.

Let $x$ be any point in $\Omega_{\lambda} \backslash \bar{G}_{\lambda}$ and let $\mathcal{C}$ be the (finite) right circular cone with vertex at $x$ and based on the $(N-1)$-dimensional ball of radius $\bar{r}$ obtained by intersecting $B$ with the hyperplane perpendicular to the vector $x-y$ and passing through $y$. Since $\Omega_{\lambda}$ is convex, then $\mathcal{C} \subset \Omega_{\lambda}$. Moreover, being $B \subset G_{\lambda}$, the axis of $\mathcal{C}$ intersects $\partial G_{\lambda}$ at a point $\zeta \notin \pi_{\lambda}$.

It is clear that the aperture $2 \theta$ of $\mathcal{C}$ is such that $\theta \geq \arctan \left(\bar{r} / d_{\Omega}\right)$, and hence (2.7) holds.

Corollary 2.3 Let $\Omega$ be a convex domain and $\partial \Omega$ be of class $C^{1}$.

Then, any maximal cap $\Omega_{\lambda}$ has a Lipschitz continuous boundary $\partial \Omega_{\lambda}$ with Lipschitz constant bounded by $2 d_{\Omega} / r_{\Omega}$.

We now state our main result.

Theorem 2.4 Let $\Omega \subset \mathbb{R}^{N}$ be a $(\mathcal{C}, \theta)$-domain with boundary of class $C^{2, \alpha}$ and let $f$ be a locally Lipschitz continuous function with $f(0) \geq 0$.

There exist two positive constants $\varepsilon$ and $C$ that depend on

$$
N, f, d_{\Omega}, r_{\Omega}, \text { the } C^{2, \alpha} \text {-regularity of } \Omega, \max _{\bar{\Omega}} u, \min _{\partial \Omega} u_{\nu},
$$

and a number $\tau \in(0,1)$ such that, if $\left[u_{\nu}\right]_{\partial \Omega} \leq \varepsilon$, then

$$
B_{r_{i}} \subset \Omega \subset B_{r_{e}},
$$

where $B_{r_{i}}$ and $B_{r_{e}}$ are two concentric balls and their radii satisfy (1.6):

$$
r_{e}-r_{i} \leq C\left[u_{v}\right]_{\partial \Omega}^{\tau}
$$


Remark 2.5 (a) As it will be clear from the proof, it holds that $\tau=1 /(1+\gamma)$ where $\gamma$ is given by (3.5); $\gamma$ depends on the half-aperture $\theta$ of the cone $\mathcal{C}$ in the definition of $(\mathcal{C}, \theta)$-domain and on the Harnack's constant for Eq. (2.5).

(b) It is clear that Theorem 1.1 is an easy corollary of this theorem, by Proposition 2.2.

(c) As explained in [1], in Theorem 2.4 some precautions are in order. The dependence of $\varepsilon$ and $C$ on a lower bound for $u_{v}$ is needed in case $f(0)=0$. In fact, if $f(0)>0$, a comparison argument in an interior ball touching $\partial \Omega$ shows that the minimum of $u_{v}$ on $\partial \Omega$ can be bounded from below by $f(0)$ times a constant that only depends on $N, \Omega,\|u\|_{\infty}$ and $f$. When $f(0)=0$, instead, the first inequality in (4.4) below does not hold and the constant $\varepsilon$ (and hence $C$ ) must depend on a lower bound for $u_{\nu}$. This fact can be seen by considering any (positive) multiple of the first Dirichlet eigenfunction $\phi_{1}$ for $-\Delta$ : in fact, for any $n \in \mathbb{N}$ the function $\phi_{1} / n$ satisfies (1.1) with $f(u)=\lambda_{1} u$, being $\lambda_{1}$ the first Dirichlet eigenvalue; although $\left(\phi_{1} / n\right)_{\nu} \rightarrow 0$ on $\partial \Omega$ as $n \rightarrow \infty$, one cannot expect to derive any information on the shape of $\Omega$.

The question whether Theorem 2.4 or [1, Theorem 1] still hold if $f(0)<0$ remains open (see [1, Section 5.1]).

The proof of Theorem 2.4 will be carried out in Sects. 3 and 4. Since it is quite long and complex, for the reader's convenience, we give an outline which consists of three main steps.

(I) We first improve [1, Proposition 1]. We fix a direction $\Omega$ and apply the procedure of moving planes. For a sufficiently small $\delta>0$, we consider the set $\Omega(\delta)$ in (2.1) and show that there exists a connected component $\Sigma_{\delta}$ of $\Omega(\delta) \cap \mathcal{H}_{\lambda}$ such that

$$
\|w\|_{L^{\infty}\left(\Sigma_{\delta}\right)} \leq \frac{C}{\delta^{\gamma}}\left[u_{\nu}\right]_{\partial \Omega}
$$

where $C$ and $\gamma$ do not depend on $\delta$ and $\Omega$ (see Proposition 4.1 below).

(II) Let $X(\delta)$ be the union of $\Sigma_{\delta}$ and its reflected image in the critical hyperplane $\pi_{\lambda}$. Since $u$ grows linearly near $\partial \Omega$, the smallness of $w$ in $\Sigma_{\delta}$ implies that $X(\delta)$ fits $\Omega$ well (see Lemma 4.3 and Proposition 4.4 below). This step gives the approximate symmetry of $\Omega$ in the direction $\Omega$.

(III) Since the arguments in (I) and (II) do not depend on the chosen direction, we apply them for $N$ (mutually) orthogonal directions and obtain a point $\mathcal{O}$ as the intersection of the corresponding $N$ critical hyperplanes. The point $\mathcal{O}$ can be chosen as an approximate center of symmetry. In fact, we are able to define two balls centered at $\mathcal{O}$ such that Theorem 2.4 holds (see the proof of Theorem 2.4 in Sect. 4 below).

We notice that once one proves the first step, (I), the remaining ones, (II) and (III), follow from a well-established argument as in [1] and [11].

\section{Harnack's inequality in a cone}

In this section, we prove some results based on Harnack-type inequality in a cone, which will be used in Sect. 4 to prove step (I) (in particular Proposition 4.1).

Let $0<a<1$ be fixed. It is well known (see [13, Theorem 8.20]) that a solution $w$ of (2.5) satisfies the following Harnack's inequality

$$
\sup _{B_{a r}} w \leq \mathfrak{H}_{a} \inf _{B_{a r}} w
$$


for any ball $B_{r} \subset \Omega_{\lambda}$; the Harnack constant $\mathfrak{H}_{a}$ can be bounded by the power $\sqrt{N}+\sqrt{r}\|c\|_{\infty}$ of a constant only depending on $N$ and $a$ (see [13]). For instance, if $c(x) \equiv 0$, by the explicit Poisson's representation formula for harmonic functions, we have that

$$
\sup _{B_{a r}} w \leq\left(\frac{1+a}{1-a}\right)^{N} \inf _{B_{a r}} w,
$$

for any $B_{r} \subset \Omega_{\lambda}$ (see [13]).

The following Lemma consists of an application of Harnack's inequality to a Harnack's chain of balls contained in a cone. The result is well known (see $[15,16,22])$; however, since we are interested in a quantitative version of it, we provide our proof.

Lemma 3.1 Fix a number $a \in(0,1)$. Pick $z \in \partial \Omega \cap \overline{\mathcal{H}}_{\lambda}$ and let $\mathcal{C}$ be any right spherical cone contained in $\Omega_{\lambda}$ and with vertex at $z$; let $2 \theta$ be the aperture of $\mathcal{C}$.

Let $w$ be given by (2.4) and pick any two points $x$ and $\xi$ on the axis of $\mathcal{C}$ such that

$$
|x-z|<|\xi-z| \text {. }
$$

Then we have that

$$
\frac{|x-z|^{\gamma}}{K} w(x) \leq w(\xi) \leq \frac{K}{|x-z|^{\gamma}} w(x),
$$

where

$$
\begin{gathered}
K=\mathfrak{H}_{a}\left[\frac{|\xi-z|(1-a \sin \theta)}{1-a}\right]^{\gamma}, \\
\gamma=\log _{\beta} \mathfrak{H}_{a}, \quad \beta=\frac{1+a \sin \theta}{1-a \sin \theta},
\end{gathered}
$$

and $\mathfrak{H}_{a}$ is given by (3.1).

Proof Here, we prove the second inequality in (3.3); the first one can be proved similarly.

Let $\ell$ be the unit vector defining the axis of $\mathcal{C}$ through $z$, that is for instance

$$
\ell=\frac{x-z}{|x-z|}
$$

We now construct a chain of balls $B_{r_{i}}\left(p_{i}\right), i=0,1, \ldots, n$, joining $x$ to $y$ with the following specifications:

(i) the centers $p_{0}, p_{1}, \ldots, p_{n}$ belong to the axis of $\mathcal{C}$;

(ii) $p_{0}=x, r_{0}=|x-z|$ and $\xi \in B_{r_{n}}\left(p_{n}\right)$;

(iii) the balls $B_{r_{1}}\left(p_{1}\right), \ldots, B_{r_{n}}\left(p_{n}\right)$ are all contained in $\mathcal{C}$ and tangent to the lateral surface of $\mathcal{C}$;

(iv) the radii $r_{1}, \ldots, r_{n}$ are chosen such that the balls $B_{a r_{0}}\left(p_{0}\right), \ldots, B_{a r_{n}}\left(p_{n}\right)$ are pairwise disjoint and

$$
\overline{B_{a r_{i}}\left(p_{i}\right)} \cap \overline{B_{a r_{i+1}}\left(p_{i+1}\right)}=\left\{p_{i}+a r_{i} \ell\right\}, \quad i=0, \ldots, n-1 .
$$

A calculation shows that (i)-(iv) determine the $r_{i}$ 's and $p_{i}$ 's as

$$
r_{i}=r_{0} \frac{(1-a) \sin \theta}{1-a \sin \theta} \beta^{i}, \quad p_{i}=z+r_{0} \frac{1-a}{1-a \sin \theta} \beta^{i} \ell, \quad i=1, \ldots, n .
$$


Since $\left|p_{n-1}-z\right| \leq|\xi-z|$, from the second formula in (3.6) we obtain a bound for $n$ :

$$
n \leq 1+\frac{\log _{\mathfrak{H}_{a}}\left(\frac{|\xi-z|}{r_{0}} \frac{1-a \sin \theta}{1-a}\right)}{\log _{\mathfrak{H}_{a}} \beta}
$$

As usual, the application of Harnack's inequality (3.1) to each ball $B_{r_{i}}\left(p_{i}\right)$ of the chain gives that $w(\xi) \leq \mathfrak{H}_{a}^{n} w(x)$; (3.3) then follows from (3.7) and some algebraic manipulations.

Remark 3.2 We notice that when $c(x) \equiv 0$ (and hence $\Delta w=0$ ), we have that

$$
\gamma=N \frac{\log \frac{1+a}{1-a}}{\log \frac{1+a \sin \theta}{1-a \sin \theta}} .
$$

We observe that $\gamma \geq N$ and equality holds only if $\theta=\pi / 2$.

\section{Stability for Serrin's problem: the proof}

In this section, thanks to the preparatory lemmas of Sect. 3 and by following the outline announced in Sect. 2, we shall bring the proof of Theorem 2.4 to an end.

As already mentioned, the crucial step in the proof is item (I) in the outline; it is the analog of [1, Proposition 1]. We modify and improve the procedure used in [1] at two salient points: (i) the definition of the approximating symmetric set $X(\delta)$ and (ii) the bound on the smallness of the function $w$ in (2.4) in terms of the parameter $\delta$ and the seminorm $\left[u_{v}\right]_{\partial \Omega}$. We draw the reader's attention on the fact that, while (i) is due to a refinement, based on Carleson-type estimates, of the technique used in [1], for (ii) the assumption that $\Omega$ is $(\mathcal{C}, \theta)$-domain is necessary - and, so far, we are not able to remove it - to treat the case of a general bounded domain with $C^{2, \alpha}$-smooth boundary. More precisely, to every maximal cap that comes about in the method of moving planes, we want to apply Lemma 3.1 that, roughly speaking, requires that such maximal cap is Lipschitz continuous, which is essentially our definition of $(\mathcal{C}, \theta)$ domain, that is certainly fulfilled if $\Omega$ is convex, as shown in Proposition 2.2. In other words, we are so far unable to exclude (even generically) that the situation depicted in Fig. 1b may occur.

We now start the procedure. For a fixed direction $\Omega$, we let $\lambda$ be the number defined in (2.3); we then consider the connected component $\Sigma$ of $\Omega_{\lambda}$ which intersects the interior touching ball at the point $P$, if case (S1) occurs, or at the point $Q$, if (S2) occurs.

For $\delta>0$, we consider the set $\Omega(\delta)$ defined in (2.1) and define $\Sigma_{\delta}$ as the connected component of $\Omega(\delta) \cap \mathcal{H}_{\lambda}$ contained in $\Sigma$-notice that our definition of $\Sigma_{\delta}$ differs from that in [1]. In addition, we fix the domain $G$ in (2.6) by choosing $t=1 / 32$ and we set $a=1 / 2$ in Lemma 3.1.

Proposition 4.1 Let $\Omega$ be a $(\mathcal{C}, \theta)$-domain.

Then, there is a constant $C=C\left(N, \Omega, d_{\Omega}, r_{\Omega}, f, \max _{\bar{\Omega}} u\right)$ such that

$$
\|w\|_{L^{\infty}\left(\Sigma_{\delta}\right)} \leq C \delta^{-\gamma}\left[u_{\nu}\right]_{\partial \Omega} \text { for } 0<\delta \leq r_{\Omega} / 32,
$$

where $\gamma$ and $\beta$ are the numbers defined in (3.5).

Proof Let $\widetilde{\Sigma}=\left\{x \in \Sigma: \operatorname{dist}(x, \partial \Sigma)>r_{\Omega} / 64\right\}$. We apply [1, Proposition 1] with $\delta=r_{\Omega} / 64$ and obtain that

$$
\|w\|_{L^{\infty}\left(\widetilde{\Sigma}_{\delta}\right)} \leq \widetilde{C}\left[u_{\nu}\right]_{\partial \Omega},
$$


for some constant $\widetilde{C}=\widetilde{C}\left(N, \Omega, d_{\Omega}, r_{\Omega}, f,\|u\|_{\infty}\right)$. By using an argument analogous to the ones in the proofs of [11, Lemmas 3.2 and 4.2] —which are based on boundary Harnack's inequality —we can extend the previous estimate to $G_{\lambda}$ :

$$
\|w\|_{L^{\infty}\left(G_{\lambda}\right)} \leq M C\left[u_{\nu}\right]_{\partial \Omega},
$$

where $M$ is the constant appearing in the boundary Harnack inequality (see Theorem 1.3 in [3]). It is important to notice that the bound in (4.2) does not depend on $\delta$.

Let $x$ be any point in $\Sigma_{\delta} \backslash \bar{G}_{\lambda}$, with $\delta<r_{\Omega} / 32$. Since $\Omega$ is a $(\mathcal{C}, \theta)$-domain, from Lemma 3.1, by choosing $\xi$ equal to the point $\zeta \in \partial G_{\lambda} \backslash \pi_{\lambda}$ (in the definition of (C, $\theta$ )-domain) in Lemma (3.1), we obtain that

$$
w(x) \leq K \delta^{-\gamma} w(\zeta)
$$

(4.2) then yields (4.1) with $C=\widetilde{C} K M$.

Remark 4.2 The statement of Proposition 4.1 differs from that of [1, Proposition 1] in three aspects: (i) the set $\Sigma_{\delta}$ we consider extends up to the hyperplane $\mathcal{H}_{\lambda}$; (ii) the dependence on $\delta$ in (4.1) greatly improves that of [1, Eq. (8)] that blows up exponentially as $\delta \rightarrow 0$; and (iii) the seminorm $\left[u_{v}\right]_{\partial \Omega}$ replaces the norm $\left\|u_{v}-d\right\|_{C^{1}(\partial \Omega)}$.

Now, we define a symmetric open set as

$$
X(\delta)=\text { the interior of } \Sigma_{\delta} \cup \Sigma_{\delta}^{\lambda} \cup\left(\partial \Sigma_{\delta} \cap \pi_{\lambda}\right) ;
$$

we want to show that $X(\delta)$ fits $\Omega$ well; how well is the main point of this paper.

The main idea is to combine the estimate (4.1) on the smallness of $w$, together with the fact the $u$ grows linearly near $\partial \Omega$; as shown in [1, Proposition 4] indeed, we know that

$$
\underline{K} \operatorname{dist}(x, \partial \Omega) \leq u(x) \leq \bar{K} \operatorname{dist}(x, \partial \Omega) \text { for all } x \in \Omega,
$$

where

$$
1 / \underline{K}, \bar{K} \leq C=C\left(\Omega, d_{\Omega}, \max _{\bar{\Omega}} u, f, \min _{\partial \Omega} u_{v}\right) .
$$

In the following lemma, we give our version [1, Eq. (34)].

Lemma 4.3 For $0<\sigma, \delta \leq r_{\Omega} / 16$ let $\Omega(\sigma)$ and $X(\delta)$ be the sets defined in (2.1) and (4.3), respectively. Let $\gamma$ and $C$ be given by (3.5) and (4.1), respectively.

If

$$
\underline{K} \sigma>C \delta^{-\gamma}\left[u_{\nu}\right]_{\partial \Omega}+\bar{K} \delta
$$

then we have that

$$
\Omega(\sigma) \subset X(\delta) \subset \Omega .
$$

Proof We have that $X(\delta) \subset \Omega$ by construction.

To show the first inclusion in (4.6), we proceed by contradiction. Since the maximal cap $\Omega_{\lambda}$ contains a ball of radius $r_{\Omega} / 4$, then $X(\delta)$ intersects $\Omega(\sigma)$. Assume that there exists a point $y \in \Omega(\sigma) \backslash X(\delta)$ and let $x$ be any point in $X(\delta) \cap \Omega(\sigma)$. Since $\Omega(\sigma)$ is connected, $x$ is joined to $y$ by a path contained in $\Omega(\sigma)$. Let $z$ be the first point on this path which falls outside $X(\delta)$. It is clear that $z \in \partial X(\delta) \cap \Omega(\sigma)$. We now consider two cases.

If $z \cdot \omega<\lambda$, then the reflection $z^{\lambda}$ of $z$ in $\pi_{\lambda}$ is such that $z^{\lambda} \in \partial \Sigma_{\delta}$ and $\operatorname{dist}\left(z^{\lambda}, \partial \Omega\right)=\delta$. Since $u(z)=w\left(z^{\lambda}\right)+u\left(z^{\lambda}\right)$, from (4.1) and (4.4) we have that

$$
\underline{K} \sigma \leq u(z) \leq C \delta^{-\gamma}\left[u_{\nu}\right]_{\partial \Omega}+\bar{K} \delta,
$$

a contradiction. 
If $z \cdot \omega \geq \lambda$, then $z \in \partial \Sigma_{\delta}$ and $\operatorname{dist}(z, \partial \Omega)=\delta$. Hence, from (4.4) we obtain that $u(z) \leq \bar{K} \delta$ and (4.7) holds as well. Since $z \in \Omega(\sigma)$, then from (4.4) we have that $u(z) \geq \underline{K} \sigma$ which contradicts (4.5) on account of (4.7).

We draw the reader's attention on the differences in (4.5) compared to [1, Eq. (34)]: (i) thanks to Lemma 3.1, the term $\delta^{-\gamma}$ replaces one in [1, Eq. (34)] that blows up exponentially; (ii) due to the different definition of the symmetrized set $X(\delta)$ (denoted by $X_{\delta}$ in [1]), we simplify the last summand in (4.5).

Proposition 4.4 Let $\gamma$ be given by (3.5). There exist positive numbers $C$ and $\varepsilon$, depending on $\Omega, d_{\Omega}, r_{\Omega}, \max _{\bar{\Omega}} u, f, \min _{\partial \Omega} u_{\nu}$, and $\sigma, \delta>0$ such that (4.6) holds with

$$
\delta<\sigma<C\left[u_{\nu}\right]_{\partial \Omega}^{\frac{1}{\gamma+1}}
$$

provided that $\left[u_{\nu}\right]_{\partial \Omega} \leq \varepsilon$.

Proof We must choose $\delta, \sigma \leq r_{\Omega} / 16$ that satisfy (4.5). If we let

$$
\delta=\left(\frac{C}{\bar{K}}\right)^{\frac{1}{\gamma+1}}\left[u_{\nu}\right]_{\partial \Omega}^{\frac{1}{\gamma+1}} \text { and } \sigma=\frac{4 \bar{K}}{\underline{K}} \delta,
$$

since $\bar{K} \delta=C \delta^{-\gamma}\left[u_{n}\right]_{\partial \Omega}$, then (4.5) holds provided that $\sigma \leq r_{\Omega} / 16$, that is for

$$
\varepsilon \leq \frac{\bar{K}}{C}\left(\frac{r_{\Omega} \underline{K}}{64 \bar{K}}\right)^{\gamma+1},
$$

The conclusion then follows from Lemma 4.3.

We are now ready to prove the main result of this paper.

Proof of Theorem 2.4 The proof is analogous to that of [1, Theorem 1]; here, we use (4.8) in place of [1, Eq. (33)].

Indeed, for any fixed direction $\omega \in \mathbb{S}^{N-1}$, Proposition 4.4 implies that if $\sigma$ satisfies (4.8), then for any $x \in \partial \Omega$ there exists $y_{\omega} \in \partial \Omega$ such that

$$
\left|x^{\lambda}-y_{\omega}\right| \leq 2 \sigma,
$$

where $x^{\lambda}$ is the reflection of $x$ in the critical hyperplane $\pi_{\lambda}$ with $\lambda$ defined by (2.3) (see also [1, Corollary 2]).

We now choose $N$ orthogonal directions, say $e_{1}, \ldots, e_{N}$, denote by $\pi_{1}, \ldots, \pi_{N}$ the corresponding critical hyperplanes and we place the origin 0 of $\mathbb{R}^{N}$ at the (unique) point in common to all the $\pi_{j}$ 's. If we denote by $R_{j}(x)$ the reflection of a point $x$ in $\pi_{j}$, we have that

$$
-x=\left(\mathcal{R}_{N} \circ \cdots \circ \mathcal{R}_{1}\right)(x)
$$

also, if we set $y_{0}=x, y_{1}=y_{e_{1}}$ and, for $j=2, \ldots, N, y_{j}$ as the point in $\partial \Omega$ such that $\left|\mathcal{R}_{j}\left(y_{j-1}\right)-y_{j}\right| \leq 2 \sigma$ determined by (4.9), we obtain that

$$
\begin{aligned}
\mid & -x-y_{N} \mid \\
& \leq\left|\left(\mathcal{R}_{N} \circ \cdots \circ \mathcal{R}_{1}\right)(x)-\mathcal{R}_{N}\left(y_{N-1}\right)\right|+\left|\mathcal{R}_{N}\left(y_{N-1}\right)-y_{N}\right| \\
& \leq\left|\left(\mathcal{R}_{N-1} \circ \cdots \circ \mathcal{R}_{1}\right)(x)-y_{N-1}\right|+2 \sigma \\
& \leq\left|\left(\mathcal{R}_{N-2} \circ \cdots \circ \mathcal{R}_{1}\right)(x)-y_{N-2}\right|+4 \sigma \leq \cdots \leq 2 N \sigma .
\end{aligned}
$$


Thus, we showed that there exists $y=y_{N} \in \partial \Omega$ such that

$$
|x+y| \leq 2 N \sigma .
$$

This fact and (4.9) imply that 0 is an approximate center of symmetry for $\Omega$, in the sense of [1, Proposition 6], that is, for any direction $\Omega$, for the critical hyperplane $\pi$ in the direction $\Omega$ we have that

$$
\operatorname{dist}(0, \pi) \leq 4 N\left[1+d_{\Omega}\right] \sigma .
$$

By letting

$$
r_{i}=\min _{x \in \partial \Omega}|x|, \quad \text { and } r_{e}=\max _{x \in \partial \Omega}|x|,
$$

we obtain (1.6) thanks to (4.10), [1, Proposition 7] and (4.8).

Notice that Theorem 2.4 was proved by fixing $a=1 / 2$ in Lemma 3.1. However, an analog of Theorem 2.4 for any fixed $a \in(0,1)$ can be proved by the same arguments and the number $\gamma$ in (3.5) and the exponent in (1.6) may be optimized in terms of $a$. It is clear that the constants $\varepsilon$ and $C$ will change accordingly (the smaller is $\gamma$, the larger is $C$ and smaller is $\varepsilon$ ). By using this plan, in case $\Omega$ is convex and $u$ is a solution of the torsional rigidity problem-i.e. when $f(u)=1$ in $(1.1)$ - we are able to give more explicit formulas and partially compare our results to those in [6].

Corollary 4.5 Let $\Omega$ be a bounded convex domain with boundary of class $C^{2, \alpha}$. Let $u$ be a solution of

$$
\Delta u=-1 \text { in } \Omega, \quad u=0 \text { on } \partial \Omega .
$$

Then, for any $\eta>0$ there exist constants $C$ and $\varepsilon$ that depend on $N, \Omega, d_{\Omega}, r_{\Omega}$ and $\eta$, such that

$$
r_{e}-r_{i} \leq C\left[u_{\nu}\right]_{\partial \Omega}^{\frac{1}{\tau+\eta}}
$$

provided that $\left[u_{\nu}\right]_{\partial \Omega} \leq \varepsilon$, where

$$
\tau=1+N \sqrt{1+\left[\frac{2 d_{\Omega}}{r_{\Omega}}\right]^{2}} .
$$

Proof We recall that, in the case in hand, $\gamma$ is given by (3.8) and is increasing as $a$ grows; thus, the optimal exponent should be looked for when $a \rightarrow 0^{+}$. Therefore, for a $(\mathcal{C}, \theta)$-domain, the exponent in (1.6) behaves as

$$
\frac{1}{1+\gamma}=\frac{1}{1+N / \sin \theta+o(1)} \quad \text { as } a \rightarrow 0^{+} .
$$

The conclusion then follows from Proposition 2.2.

We finally notice that, in this case, the dependence of the constants $C$ and $\varepsilon$ appearing in Theorem 2.4 on the quantities $\|u\|_{\infty}$ and $\min _{\partial \Omega} u_{v}$ ) can be removed, since these quantities can be bounded in terms of the $C^{2}$ regularity of $\Omega$, by standard barrier techniques.

Remark 4.6 In [6, Theorem 2] the authors prove an estimate similar to (4.11). When $\Omega$ is convex, our estimate improves that in [6, Theorem 2] if

$$
\frac{d_{\Omega}}{r_{\Omega}} \leq \sqrt{\frac{2 N^{2}+N-5 / 2}{N}} .
$$


Acknowledgments The authors wish to thank Paolo Salani for the useful discussions they had together. The authors have been supported by the Gruppo Nazionale per l'Analisi Matematica, la Probabilità e le loro Applicazioni (GNAMPA) of the Istituto Nazionale di Alta Matematica (INdAM).

The paper was completed while the author was visiting "The Institute for Computational Engineering and Sciences" (ICES) of The University of Texas at Austin, and he wishes to thank the Institute for hospitality and support. The author has been also supported by the NSF-DMS Grant 1361122 and the Firb project 2013 "Geometrical and Qualitative aspects of PDE".

\section{References}

1. Aftalion, A., Busca, J., Reichel, W.: Approximate radial symmetry for overdetermined boundary value problems. Adv. Differ. Equ. 4, 907-932 (1999)

2. Aleksandrov, A.D.: Uniqueness theorems for surfaces in the large V. Vestnik Leningrad Univ. 13, 5-8 (1958) [English translation: Am. Math. Soc. Transl. Ser. 21(2), 412-415 (1962)]

3. Berestycki, H., Caffarelli, L.A., Nirenberg, L.: Inequalities for second-order elliptic equations with applications to unbounded domains I. Duke Math. J. 81(2), 467-494 (1996)

4. Berestycki, H., Nirenberg, L., Varadhan, S.R.S.: The principal eigenvalue and maximum principle for second-order elliptic operators in general domains. Commun. Pure Appl. Math. 47, 4792 (1994)

5. Brandolini, B., Nitsch, C., Salani, P., Trombetti, C.: Serrin type overdetermined problems: an alternative proof. Arch. Ration. Mech. Anal. 190, 267-280 (2008)

6. Brandolini, B., Nitsch, C., Salani, P., Trombetti, C.: On the stability of the Serrin problem. J. Differ. Equ. 245, 1566-1583 (2008)

7. Brandolini, B., Nitsch, C., Salani, P., Trombetti, C.: Stability of radial symmetry for a Monge-Ampère overdetermined problem. Ann. Math. Pura Appl. 188(3), 445-453 (2009)

8. Chayes, L., González, M., Guandani, M., Kim, I.C.: Global existence and uniqueness of solutions to a model of price formation. SIAM J. Math. Anal. 41(5), 2107-2135 (2009)

9. Ciraolo, G., Magnanini, R.: A note on Serrin's overdetermined problem. Kodai Math. J. 37, 728-736 (2014)

10. Ciraolo, G., Magnanini, R., Sakaguchi, S.: Symmetry of solutions of elliptic and parabolic equations with a level surface parallel to the boundary, to appear in J. Eur. Math. Soc. (JEMS). Preprint arXiv:1203.5295

11. Ciraolo, G., Magnanini, R., Sakaguchi, S.: Solutions of elliptic equations with a level surface parallel to the boundary: stability of the radial configuration, to appear in J. Anal. Math. Preprint arXiv:1307.1257

12. Fraenkel, L.E.: An Introduction to Maximum Principles and Symmetry in Elliptic Problems. Cambridge University Press, Cambridge (2000)

13. Gilbarg, D., Trudinger, N.S.: Elliptic Partial Differential Equations of Second Order, 2nd edn. Springer, Berlin (1983)

14. Henrot, A.: Extremum Problems for Eigenvalues of Elliptic Operators. Birkäuser, Basel (2006)

15. Jerison, D., Konig, C.: Boundary behavior of harmonic functions in nontangentially accessible domains. Adv. Math. 46, 80-147 (1982)

16. Konig, C.: Harmonic analysis techniques for second order elliptic boundary value problems. CBMS Regional Conf. Series in Math., no. 83, Amer. Math. Soc., Providence, RI (1994)

17. Lasry, J.M., Lions, P.L.: Mean field games. Jpn. J. Math. 2(1), 229-260 (2007)

18. Magnanini, R., Sakaguchi, S.: Matzoh ball soup: heat conductors with a stationary isothermic surface. Ann. Math. 156, 931-946 (2002)

19. Magnanini, R., Sakaguchi, S.: Nonlinear diffusion with a bounded stationary level surface. Ann. Inst. H. Poincaré Anal. Non Linéaire 27, 937-952 (2010)

20. Magnanini, R., Sakaguchi, S.: Matzoh ball soup revisited: the boundary regularity issue. Math. Methods Appl. Sci. 36, 2023-2032 (2013)

21. Markowich, P.A., Matevosyan, N., Pietschmann, J.F., Wolfram, M.T.: On a parabolic free boundary equation modelling price formation. Math. Models Methods Appl. Sci. 19(10), 1929-1957 (2009)

22. Moser, J.: A Harnack inequality for parabolic differential equations. Commun. Pure Appl. Math. 17, 101134 (1964)

23. Payne, L.E., Schaefer, P.W.: Duality theorems in some overdetermined boundary value problems. Math. Methods Appl. Sci. 11, 805-819 (1989)

24. Serrin, J.: A symmetry problem in potential theory. Arch. Ration. Mech. Anal. 43, 304-318 (1971)

25. Shahgholian, H.: Diversifications of Serrin's and related symmetry problems. Complex Var. Elliptic Equ. 57, 653-665 (2012)

26. Weinberger, H.F.: Remark on the preceding paper of Serrin. Arch. Ration. Mech. Anal. 43, 319-320 (1971) 